\title{
Colorectal anastomotic leak: delay in reintervention after false- negative computed tomography scan is a reason for concern
}

\author{
C. C. M. Marres ${ }^{1}$ A. W. H. van de Ven ${ }^{1,2}$ - L. G. J. Leijssen ${ }^{1}$ - P. C. M. Verbeek ${ }^{1}$ \\ W. A. Bemelman ${ }^{2}$ C. J. Buskens ${ }^{2}$
}

Received: 21 March 2017/Accepted: 1 September 2017/Published online: 19 September 2017

(c) The Author(s) 2017. This article is an open access publication

\begin{abstract}
Background Early detection of anastomotic leakage (AL) after colorectal surgery followed by timely reintervention is of crucial importance. The aim of this study was to investigate the accuracy of computed tomography (CT) imaging for $\mathrm{AL}$ and the effects of delay in reintervention after a false-negative CT.

Methods All files from patients who had colorectal surgery with primary anastomoses between 2009 and 2014 were reviewed. The predictive value of $\mathrm{CT}$ scanning for AL was determined and correlated with short-term postoperative patient outcomes. In addition, factors predictive of falsenegative scans were assessed.

Results Six hundred and twenty-eight patient files were reviewed. In total, a CT scan was performed in 127 patients. Overall, leakage was seen in 49 patients $(7.8 \%)$. The positive and negative predictive values were 78 and $88 \%$, respectively. Sensitivity was $73 \%$ and specificity $91 \%$. In patients with a true-positive CT $(n=24)$, reintervention followed after a median interval of 0 days (IQR 1 ), whereas this was 1 day (IQR 2 ) in the false-negative group $(n=11)(p<0.05)$. This was associated with a significantly increased mortality rate $(1 / 24=4.2 \%$ vs $5 / 11=45.5 \%)(p<0.005)$, an increased length of hospital stay [median 28 days (IQR 26) vs 54 days (IQR 20) $(p<0.05)]$.
\end{abstract}

C. C. M. Marres

cmarres@gmail.com

1 Department of Surgery, Flevo Hospital, Hospitaalweg 1, 1315 RA Almere, The Netherlands

2 Department of Surgery, Academical Medical Center, Amsterdam, The Netherlands
Conclusions Delayed reintervention after false-negative CT scanning is associated with a high mortality rate and a significant increase in length of hospital stay.

Keywords Colorectal surgery - Computed tomography · Anastomotic leakage · Oncology

\section{Introduction}

Anastomotic leakage (AL) is a life-threatening complication after colorectal surgery [1]. In the literature, mortality rates after AL vary from 15 to $33 \%$. Early detection of AL followed by timely reintervention decreases mortality rates. In daily clinical practice, abdominal computed tomography (CT) scanning is most frequently used to diagnose or exclude $\mathrm{AL}$ after colorectal surgery [2-4]. Compared to water-soluble contrast enema and plain X-ray, CT scan is more sensitive and is able to detect other complications such as bleeding, perforation or abscess. At the same time, it can be used to guide therapeutic percutaneous drainage of abscesses.

Considering how commonly CT is performed in postoperative colorectal patients, the literature on the accuracy of CT scanning in patients with AL is scarce. The only systematic review, performed by Kornmann et al. [5], included a total of only 221 abdominal CT scans from eight different studies. This review showed a relatively low sensitivity of $68 \%$, with a large range reported from these small, retrospective studies. The technical quality of the CT scan was often suboptimal (10-mm sections, no enteral or intravenous contrast), and the definition of $\mathrm{AL}$ was inconsistent. Previous studies suggested that false-negative CT outcome may delay reintervention $[6,7]$, but the clinical consequences of a false-negative $\mathrm{CT}$ scan were only described in one study [8]. 
Recent studies by Huiberts et al. [9] and Kauv et al. [10] showed that leakage of contrast medium was the only independent predictive factor for AL. In our institution, abdominal CT scanning with rectal contrast enema (RCE) in cases where there is suspicion of $\mathrm{AL}$ has been the standard imaging procedure for over 6 years.

The aim of this study was to investigate the accuracy of abdominal CT scanning with RCE for anastomotic leakage and the effect of false-negative scans on delay in therapeutic intervention and clinical outcome.

\section{Materials and methods}

Data from a prospectively maintained database of all patients who had elective or emergency colorectal surgery with primary anastomoses for malignant or benign disease between 2009 and 2014 were reviewed. Patient characteristics, including age, gender and American Society of Anesthesiologists (ASA) classification, were collated. Type of operation, complications, length of hospital stay, length of stay in the intensive care unit (ICU), indications for abdominal CT scans, timing and outcome of CT scans and subsequent reinterventions were also evaluated.

All colorectal resections were performed by, or under supervision of, a specialist colorectal surgeon. Perioperatively, patients were managed according to standard fasttrack protocol [11]. Abdominal CT was performed when patients had signs of sepsis with clinical symptoms and/or physiological deterioration (e.g. deviations in respiratory rate, pulse rate, blood pressure, temperature, urine production or neurological status).

CT imaging was performed on a 16- and 64-slicedMDCT scanner (Philips, Netherlands), with a slice thickness of algorithm of $3-5 \mathrm{~mm}$ with axial and coronal reconstructions. Scanning protocol included intravenous and rectal contrast. CT scans were reviewed by experienced radiologists. For this study, radiology reports were used as given at the time rather than later amendments, in order to evaluate the effect of delay in reintervention after false-negative scans. Our radiologists scored the following features to asses the CT scans: fluid intraabdominally, fluid near the anastomosis, free air in the abdomen, air near the anastomosis and contrast from the lumen. AL was graded according to the definition of the International Study Group of Rectal Cancer [12]. Grade C was defined as a leak requiring surgical reintervention, grade $\mathrm{B}$ as a leak requiring percutaneous reintervention, and grade $\mathrm{A}$ as a leak requiring antibiotics at the most. Since the indication for antibiotics was not always based on CT scan findings and it had minimal to no clinical impact on the patients postoperative course, only grade $\mathrm{B}$ and $\mathrm{C}$ anastomotic leaks were included in the CT accuracy analyses.

\section{Statistical analysis}

Statistical analysis was performed using SPSS software, version 22.0 (SPSS Inc, Chicago, IL, USA). Sensitivity, specificity, positive predictive value (PPV) and negative predictive value (NPV) were calculated with $95 \%$ confidence intervals (CI). Categorical data are presented as frequencies and percentages compared by the Chi-square test. The parametric and nonparametric continuous data are presented as means and standard deviations and were analysed by the Mann-Whitney $U$ test. Missing data for every variable were less than $10 \%$, and therefore there no imputation of missing data was performed. A two-tailed $p$ value of $<0.05$ was considered statistically significant.

\section{Results}

Between 2009 and 2014, 628 patients underwent colorectal surgery with primary anastomosis in our institution. In 127 out of 628 patients, an abdominal CT scan was performed based on clinical symptoms and/or signs of sepsis. Of these patients, $69(54.3 \%)$ were men and $58(45.7 \%)$ women with a median age of 66 years (range 44-89 years).

Ninety-nine of the 127 patients undergoing CT scan for suspicion of $\mathrm{AL}$ were given enteral contrast according to protocol (78\%). In 85 patients $(86 \%)$, the contrast reached the anastomosis. Relevant baseline characteristics are summarised in Table 1.

\section{Anastomotic leakage}

Overall leakage after surgery (grade A, B and C) was 7.8\% (49 out of 628 anastomoses). Thirty-eight patients had grade C AL (6.0\%), six patients (1.0\%) had grade B leakage and five patients had grade A leakage $(0.8 \%)$. Leak rates were comparable for patients with open or laparoscopic procedures and for patients with benign or malignant disease.

In nine patients with $\mathrm{AL}$, no $\mathrm{CT}$ was performed prior to reintervention. Six of these patients were reoperated on within 5 days of primary resection without diagnostic imaging because of their clinical condition. In the other three patients, a water-soluble contrast enema with X-ray was performed, during the implementation phase of the abdominal CT scan with contrast enema in 2009.

\section{Predictive value of the $\mathrm{CT}$ scan}

Twenty-four patients had a true-positive CT scan, with AL demonstrated at reintervention. Eleven patients had a false-negative scan; there were no signs of $\mathrm{AL}$ on $\mathrm{CT}$ scan, but a leak was confirmed afterwards during reintervention or autopsy. Seventy-nine patients had a true- 
Table 1 Demographic characteristics

\begin{tabular}{|c|c|c|}
\hline Variables & All patients $N(\%)^{\mathrm{a}}$ & Patients with CT $N(\%)^{\mathrm{a}}$ \\
\hline \multicolumn{3}{|l|}{ Age, years } \\
\hline Median & 66 & 66 \\
\hline Range & $18-96$ & $44-89$ \\
\hline \multicolumn{3}{|l|}{ Sex } \\
\hline Female & $295(47.0)$ & $58(45.7)$ \\
\hline Male & $333(53.0)$ & $69(54.3)$ \\
\hline \multicolumn{3}{|l|}{ ASA class } \\
\hline ASA 1 or 2 & $489(77.8)$ & $92(72.4)$ \\
\hline ASA 3 or 4 & $132(21.0)$ & $26(20.5)$ \\
\hline \multicolumn{3}{|l|}{ Type of operation } \\
\hline Right colectomy & $203(32.3)$ & $39(30.7)$ \\
\hline Left colectomy & $41(6.5)$ & $11(8.7)$ \\
\hline Sigmoidectomy/LAR & $310(49.4)$ & $53(41.7)$ \\
\hline Colectomy & $37(5.9)$ & $13(10.2)$ \\
\hline Other & $36(5.8)$ & $11(8.7)$ \\
\hline \multicolumn{3}{|l|}{ Open/laparoscopic } \\
\hline Open & $84(13.4)$ & $40(31.5)$ \\
\hline Laparoscopic & $512(81.5)$ & $87(68.5)$ \\
\hline \multicolumn{3}{|l|}{ Stoma } \\
\hline No stoma & $413(65.8)$ & $88(69.3)$ \\
\hline Loop ileostomy & $126(20.1)$ & $37(29.1)$ \\
\hline End colostomy & $5(0.8)$ & $2(1.6)$ \\
\hline \multicolumn{3}{|l|}{ Urgency } \\
\hline Elective & $508(80.9)$ & $93(71.9)$ \\
\hline Emergency & 87 (13.9) & $29(22.70$ \\
\hline \multicolumn{3}{|l|}{ Anastomotic leak } \\
\hline Grade C & $38(6.1)$ & $29(22.8)$ \\
\hline Grade B & $6(0.8)$ & $6(4.7)$ \\
\hline Grade A & $5(1.0)$ & $5(3.9)$ \\
\hline Total & $628(100)$ & $127(100)$ \\
\hline
\end{tabular}

$C T$ computed tomography, ASA American Society of Anesthesiologists, LAR low anterior resection

${ }^{a}$ Unless stated otherwise in the first column negative scan, and eight patients had a false-positive scan. CT scans were counted as false-positive when the scan was reported as showing signs suggestive of AL; however, either during relaparoscopy there was no leakage detected or they had no clinical signs of AL and recovered without any treatment (no antibiotics). In six patients, CT scan revealed too much free air or fluid in the abdomen (based on how much free air or fluid was expected on the corresponding day postoperatively). In one of these patients, relaparoscopy did not show any abnormality. In two patients, minimal contrast was seen near the anastomosis, but the clinical sign was absent and they recovered without any treatment.

The accuracy of CT scanning for AL in this study was $85.0 \%$. The positive and negative predictive values were, respectively, 0.78 (CI 0.65-0.92) and 0.88 (CI 0.82-0.95).
The sensitivity was 0.72 (CI $0.59-0.86)$ and specificity 0.91 (CI 0.85-0.97) (Table 2). The area under the receiver operating characteristic (ROC) curve was 0.80 . This was calculated in accordance with the recommendations of Castor et al. [13] for binary diagnostic tests.

\section{Impact on clinical outcome}

The overall mortality rate after grade $\mathrm{B}$ and $\mathrm{C}$ leakage was $20.5 \%$ (9 out of 44 patients). Out of 24 patients with a truepositive CT scan, only one died (4.2\%), whereas mortality after a false-negative CT was $45.5 \%$ (5 out of 11 patients) $(p<0.005)$. In patients with leakage predicted by CT, reintervention was performed after a median interval of 0 days (IQR 1 ), whereas this was 1 day (IQR 2) in the false-negative group $(p<0.05)$. This resulted in a 
Table 2 Sensitivity, specificity, positive and negative predictive value for anastomotic leakage

\begin{tabular}{llll}
\hline CT outcome & Anastomotic leakage & No anastomotic leakage & Results $(95 \%$ CI $)$ \\
\hline Positive & 24 & 8 & Sensitivity 0.69 (0.51-0.83) \\
& \multirow{2}{*}{ Negative } & 79 & Specificity 0.91 (0.83-0.96) \\
& & & PPV 0.75 (0.56-0.87) \\
& & NPV $0.88(0.79-0.94)$ \\
\hline
\end{tabular}

Patients with grade A anastomotic leak were excluded

Values are given as number of patients (n). Sensitivity, specificity, PPV and NPV are given with 95\% CI $C T$ computed tomography, $P P V$ positive predictive value, $N P V$ negative predictive value

Table 3 Clinical outcome of anastomotic leakage in patients with grade B and C leakage $(n=35)$

\begin{tabular}{|c|c|c|c|c|}
\hline & Overall $N=35$ & True-positive CT $N=24$ & False-negative CT $N=11$ & $p$ value \\
\hline Mortality (\%) & $6(17.1 \%)$ & $1(4.2 \%)$ & $5(45.5 \%)$ & $.003 *$ \\
\hline Length of hospital stay, median (IQR) & $30.5(31)$ & $28(26)$ & $54(20)$ & $.014 * *$ \\
\hline Days in ICU, median (IQR) & $3(10)$ & $3(10)$ & $2(14)$ & $.094 * *$ \\
\hline Days from operation to $\mathrm{CT}$, median (IQR) & $7(5)$ & $7(4)$ & $4(4)$ & $.121 * *$ \\
\hline Days from CT to reintervention, median (IQR) & $0(1)$ & $0(1)$ & $1(2)$ & $.011 * *$ \\
\hline
\end{tabular}

$C T$ computed tomography scan, $I C U$ intensive care unit, $I Q R$ interquartile range

* Chi-square test

** Mann-Whitney $U$ test

Table 4 Parameters predicting accuracy of CT scanning for anastomotic leakage

\begin{tabular}{llclll}
\hline & Overall $N=35$ & True-positive CT $N=24$ & False-negative CT $N=11$ & $p$ value \\
\hline Emergency surgery & $11(31.4 \%)$ & $7(41.2 \%)$ & $4(36.4 \%)$ & $3(27.2 \%)$ & $.670 *$ \\
Rectal anastomosis & $12(34.2 \%)$ & $9(37.5 \%)$ & $9(81.8 \%)$ & $.554^{*}$ \\
Rectal contrast & $29(82.9 \%)$ & $20(83.2 \%)$ & $6(66.6 \%)$ & $.466^{*}$ \\
Contrast reached anastomosis & $23(79.3 \%)$ & $17(85.0 \%)$ & $.874 *$ \\
\hline
\end{tabular}

CT computed tomography

* Chi-square test

significantly increased length of hospital stay [median of 28 days (IQR 26) vs 54 days (IQR 20) $(p<0.05)$ ]. The median length of stay in the ICU was 3 days (IQR 10) for patients with a true-positive CT scan versus 2 days (IQR 14) in patients with a false-negative scan (Table 3 ).

To identify predictive factors influencing CT accuracy, the following variables were analysed: anatomical site of the anastomosis, emergency surgery, whether enteral contrast was given and if the contrast had reached the anastomosis on CT scan.

There were no statistically significant differences found in these variables between patients with a true-positive and a false-negative scan. It is noteworthy, however, that the contrast reached the level of the anastomosis in only $66.6 \%$ in the false-negative group, but in $85.0 \%$ in the true-positive group (Table 4).

\section{Discussion}

This study demonstrated an $84 \%$ accuracy of CT scan for AL after colorectal surgery. A leak was missed in 11/39 patients resulting in a mediocre sensitivity of $72 \%$. Falsenegative CT was associated with a significantly higher mortality.

The sensitivity and specificity of CT scanning found in this study are in line with the results of the systematic review of Kornmann et al. [5] and with three studies on sensitivity of CT scanning [8-10].

Previous studies also showed that false-negative CT scan causes delay in reintervention after $\mathrm{AL}$, but numbers of patients are small in these reports. Kornmann et al. [8] reported that mortality increased to $63 \%$ after delayed diagnosis compared to $7 \%$ in patients with a true-positive 
CT outcome and immediate intervention, which is comparable to our results (45.5 vs $4.2 \%$ ).

The two most recent studies found that leakage of contrast medium was the only independent predictive factor for AL $[9,10]$. Kauv et al. [10] reported that 58 patients were scanned with RCE and 95 without. Of the 11 falsenegative or indeterminate $\mathrm{CT}$ scans in their study only 2 (18\%) were performed with RCE. Huiberts et al. [9] reported that RCE was given to 52 patients and 52 patients received oral contrast only. The contrast reached the anastomosis in only $31 \%$ of the patients. In the false-negative group, contrast was present at the site of the anastomosis in $39 \%$ of the cases. In our study, 99 patients were given rectal contrast. In 85 patients, the contrast reached the anastomosis $(86 \%)$. The contrast did not reach the anastomosis in $1 / 3$ of the patients with a false-negative scan.

Although several studies mention that performing a scan too early in the course of AL, i.e. before AL is radiologically detectable, leads to false-negative scans, $[6,7,14]$, in the present study no significant difference was found in the timing of the CT scans of patients with a true-positive and patients with false-negative scan.

A limitation of this study is the fact that the indications for CT scanning, timing of the intervention or "wait and see" policy are subject to a surgeon's personal opinion or experience. The only published prospective study on this subject to date suggested that abdominal complications cannot be predicted by a CT scan on day 5 after laparoscopic colorectal resection and therefore it cannot be recommended for routine use [15].

In previous studies, different definitions of clinical and radiological AL are used. Surprisingly, there is little consensus on the definition of AL, different criteria making interpretation of data more difficult $[16,17]$. The definition and grading of AL by the International Study Group of Rectal Cancer [18] were used in this study and should perhaps be used as a standard in future reports.

The strength of this study is the consecutive series from a single, nonacademic, secondary care centre, reflecting daily clinical practice of most colorectal surgeons. To our knowledge, this is the first study with detailed information on clinical outcome after false-negative CT scans. Furthermore, this is one of the largest studies on accuracy of CT scanning for AL with the largest population of patients screened with RCE.

\section{Conclusions}

Our results indicate that a false-negative CT scan in suspected AL is associated with a higher mortality rate and a significant prolonged length of hospital stay. CT scan is not accurate enough to provide assurance of anastomotic integrity and should be considered in the round with other patient parameters with a low threshold for intervention maintained if a negative scan does not fit with symptoms, signs and other results. Diagnostic laparoscopy despite a negative scan should still be considered where there remains clinical suspicion of AL.

\section{Compliance with ethical standards}

Conflict of interest The authors declare that they have no conflict of interest.

Ethical approval This article does not contain any studies with human participants or animals performed by the authors.

Informed consent For this type of study formal consent is not required.

Open Access This article is distributed under the terms of the Creative Commons Attribution 4.0 International License (http://crea tivecommons.org/licenses/by/4.0/), which permits unrestricted use, distribution, and reproduction in any medium, provided you give appropriate credit to the original author(s) and the source, provide a link to the Creative Commons license, and indicate if changes were made.

\section{References}

1. Alves A, Panis Y, Pocard M, Regimbeau JM, Valleur P (1999) Management of anastomotic leakage after nondiverted large bowel resection. J Am Coll Surg 189:554-559

2. McDermott FD, Heeney A, Kelly ME, Steele RJ, Carlson GL, Winter DC (2015) Systematic review of preoperative, intraoperative and postoperative risk factors for colorectal anastomotic leaks. Br J Surg 102(5):462-479

3. Daams F, Wu Z, Lahaye MJ, Jeekel J, Lange JF (2014) Prediction and diagnosis of colorectal anastomotic leakage: a systematic review of literature. World J Gastrointest Surg 6(2):14-26

4. Hirst NA, Tiernan JP, Millner PA, Jayne DG (2014) Systematic review of methods to predict and detect anastomotic leakage in colorectal surgery. Colorectal Dis 16(2):95-109

5. Kornmann VNN, Treskes N, Hoonhout LHF et al (2012) Systematic review on the value of CT scanning in the diagnosis of anastomotic leakage after colorectal surgery. Int J Color Dis 28(4):437-445

6. Doeksen A, Tanis PJ, Vrouenraets BC, van Lanschot JJ, van Tets WF (2007) Factors determining delay in relaparotomy for anastomotic leakage after colorectal resection. World J Gastroenterol 13(27):3721-3725

7. Khoury W, Ben-Yehuda A, Ben-Haim M et al (2009) Abdominal computed tomography for diagnosing postoperative lower gastrointestinal tract leaks. J Gastrointest Surg 13:1454-1458

8. Kornmann VN, van Ramshorst B, Smits AB, Bollen TL, Boerma D (2014) Beware of false-negative CT scan for anastomotic leakage after colonic surgery. Int J Colorectal Dis 29(4):445-451

9. Huiberts AA, Dijksman LM, Boer SA, Krul EJ, Peringa J, Donkervoort SC (2015) Contrast medium at the site of the anastomosis is crucial in detecting anastomotic leakage with CT imaging after colorectal surgery. Int J Colorectal Dis 30(6):843-848

10. Kauv P, Benadjaoud S, Curis E, Boulay-Coletta I, Loriau J, Zins M (2015) Anastomotic leakage after colorectal surgery: diagnostic accuracy of CT. Eur Radiol 25(12):3543-3551 
11. Kehlet H, Wilmore DW (2008) Evidence-based surgical care and the evolution of fast-track surgery. Ann Surg 248:189-198

12. Rahbari NN, Weitz J, Hohenberger W et al (2010) Definition and grading of anastomotic leakage following anterior resection of the rectum: a proposal by the International Study Group of Rectal Cancer. Surgery 147(3):339-351

13. Cantor SB, Kattan MW (2000) Determining the area under the ROC curve for a binary diagnostic test. Med Decis Mak 20(4):468-470

14. DuBrow RA, David CL, Curley SA (1995) Anastomotic leaks after low anterior resection for rectal carcinoma: evaluation with CT and barium enema. AJR Am J Roentgenol 165(3):567-571

15. Wagner M, Zappa M, Maggiori L, Bretagnol F, Vilgrain V, Panis Y (2014) Can postoperative complications be predicted by a routine CT scan on day 5? A study of 78 laparoscopic colorectal resections. Tech Coloproctol 18(3):239-245

16. Nicksa GA, Dring RV, Johnson KH et al (2007) Anastomotic leaks: what is the best diagnostic imaging study? Dis Colon Rectum 50:197-203

17. Adams K, Papagrigoriadis S (2013) Little consensus in either definition or diagnosis of a lower gastro-intestinal anastomotic leak amongst colorectal surgeons. Int $\mathbf{J}$ Colorectal Dis 28:967-971

18. Kulu Y, Ulrich A, Bruckner T et al (2013) Validation of the International Study Group of Rectal Cancer definition and severity grading of anastomotic leakage. Surgery 153(6):753-761 